www.jmscr.igmpublication.org

Index Copernicus Value: 79.54

ISSN (e)-2347-176x ISSN (p) 2455-0450

crossrefDOI: https://dx.doi.org/10.18535/jmscr/v7i1.120

\title{
Association of Periodontitis in patients with Coronary Artery Disease at Tertiary Care Centre of Central India
}

\author{
Authors \\ Dr Parul Jain ${ }^{1}$ (Assoc. Prof.), Dr Ashish Kumar Jain ${ }^{2}$ (Assoc. Prof.)* \\ ${ }^{1}$ Modern Dental College \& Research Centre, Gandhi Nagar, Airport Road, Indore \\ ${ }^{2}$ Dept. of Cardiology, Mahatma Gandhi Memorial Medical College, Indore \\ *Corresponding Author \\ Dr Ashish Kumar Jain
}

Assoc. Prof., Dept. of Cardiology, Mahatma Gandhi Memorial Medical College, Indore, MP, India

\begin{abstract}
Background: The aim of this study was to evaluate the association of periodontitis \& the extent \& degree of coronary artery stenosis in a Tertiary Care Centre of Central India.

Methods: Patients $(n=350)$ with coronary artery stenosis confirmed by coronary angiography were eligible to take part in the study. Only subjects that were $\geq 60$ years old, had $\geq 50 \%$ stenosis in at least one coronary artery, and did not have a history of smoking were included. After periodontal examination, probing depth calculus index, plaque index, including bleeding index and periodontal attachment loss, four groups were defined based on the severity of periodontitis: no periodontitis/gingivitis (M0, $n=68)$; mild periodontitis $(M 1, n=84)$; moderate periodontitis $(M 2, n=92)$; and severe periodontitis $(M 3, n=106)$. Extent \& and degree of coronary artery stenosis was calculated by Angiography.

Results: The Angiography Finding was significantly greater in the M3 group compared with the MO group. Multiple linear regression analysis revealed that probing depth and plaque index were significantly correlated with each other.

Conclusions: The extent \& severity of coronary artery stenosis in patients $\geq 60$ years old is positively correlated with the severity of periodontitis.

Study Design: Retrospective Study.

Keywords: Coronary artery disease, coronary artery stenosis, periodontitis, clinical attachment loss.
\end{abstract}

\section{Introduction}

Coronary heart disease (CAD) is a life-threatening disease that has a serious impact on physical and emotional well-being. CAD is also one of the major causes of death worldwide. In-depth studies on the aetiology, pathogenesis and clinical treatment of CAD have identified other important pathogenic factors in addition to the traditional risk factors of smoking, hypertension and dyslipidaemia. ${ }^{1}$ Since the 1990 s, researchers have provided evidence that infection is one of the risk factors for atherosclerosis and thrombus formation and that systemic inflammation due to infection promotes and accelerates the initiation and progression of atherosclerosis. ${ }^{2}$ There are several similarities between CAD and periodontitis.

Pathological studies of atherosclerotic plaques have identified the presence of periodontal 
pathogens. Although not all studies have shown a significant association between periodontitis and CAD, the majority of studies, as summarized in a meta-analysis by Bahekar et al., ${ }^{3}$ demonstrated a significant association between periodontal disease and CAD even after adjusting for traditional risk factors such as smoking, blood lipids, race, gender and obesity.

Infection \& inflammation are universal mechanisms in both diseases. Periodontitis is one of the main causes of tooth loss in humans. As in India tobacoo chewing, gutka chewing with lime are very common also poor oral hygine are common that leads to recurrent periodontitist, Recurrent infection of the periodontium results in higher levels of inflammatory factors in the serum. As the severity of periodontitis increases ${ }^{4}$, there is a greater systemic inflammatory response. Previous studies showed that periodontitis leads to atherosclerosis through periodontal inflammation different pathogens and their by-products, or through the release of inflammatory mediators from periodontal tissue are also responsible for it this induces endothelial dysfunction.

A limitation of previous studies that investigated the relationship between periodontal disease and CAD was that the definition of periodontitis was highly variable and not always based on a clinical examination. With coronary stenosis $(\geq 50 \%)$ documented by CAG. Use of Gensini method, ${ }^{5}$ to calculate the extent \& severity of coronary artery stenosis throughout the entire coronary arterial tree.

\section{Materials and Methods}

There were 350 patients were included in the study with CAD disease who were admitted to our hospital from January 2017 to February 2018. With the consent of the patient to undergo this study. All patients that were included in the study met the following inclusion criteria: age $\geq 60$ years old, stenosis $>50 \%$ in at least one branch of a major coronary artery confirmed by CAG \& at least 14 natural teeth in full denture. Patients were excluded if they met any of the following criteria: periodontal treatment; antibiotic administration, or medications inducing gingival overgrowth within six months of the study; pregnancy; a history of smoking or alcoholism; acute cerebrovascular disease, peripheral vascular disease or severe infection; systemic diseases including respiratory diseases, malignant tumours, or liver or kidney dysfunction \& HIV.

\section{Diagnosis of CAD}

CAD was diagnosed by CAG using Judkin's technique with projections in multiple views. All angiograms were evaluated by experienced physicians from visual assessment, and the degree of coronary stenosis was determined. The extent and severity of coronary artery stenosis was based on the Angiography. To obtain the Angiography Finding, the coronary arterial tree was divided into 14 segments, and the degree of stenosis evaluated in each segment. Score of 1 was given for 1 to $25 \%$ stenosis, 2 for 26 to $50 \%$ stenosis, 4 for 51 to $75 \%$ stenosis, 8 for 76 to $90 \%$ stenosis, 16 for 91 to $99 \%$ and 32 for total occlusion. Each segment score of was then multiplied by a weighting factor that represented the importance of a lesion in that segment. The weighting factor was 5 for a left main lesion; 2.5 for a proximal left anterior descending (LAD) or left circumflex (LCX) lesion; 1.5 for a midsegment LAD or LCX lesion, 1 for a lesion in the distal LAD, LCx, first diagonal branch, first obtuse marginal branch, right coronary artery, posterior descending artery or septal artery; and 0.5 for a lesion in the second diagonal or obtuse marginal branches. The products in each of the 14 segments were then summed to obtain the final Angiography Finding. The Angiography Finding values ranged from 2 to 190. A higher Angiography Finding indicated a greater extent and severity of coronary stenosis. Diagnosis of periodontitis According to the diagnostic standard from Armitage, 22 the grouping of periodontitis severity was based on clinical attachment loss (CAL) to determine severity. In the group with no periodontitis/ gingivitis (M0), the average CAL was $\leq 0.5 \mathrm{~mm}$, 
the number of loci (with proximal $\mathrm{CAL} \geq 3 \mathrm{~mm}$ ) was zero and the number of teeth lost was $\leq 2$ (excluding the third molar, orthodontic teeth, tooth extraction due to trauma or injury, tooth loss due to severe caries and congenital tooth loss). In the group with mild periodontitis (M1), the average CAL $\geq 0.6 \mathrm{~mm}$ but $\leq 1.5 \mathrm{~mm}$, the number of loci (with proximal $\mathrm{CAL} \geq 3 \mathrm{~mm}$ ) was zero, and the number of teeth lost was $\leq 3$. In the moderate periodontitis group (M2), the average CAL was $\geq 1.6$ but $\leq 2.4 \mathrm{~mm}$, loci (with proximal $\mathrm{CAL} \geq 3$ $\mathrm{mm}$ ) were distributed in three different areas or the total number was $\geq 6$ and the number of teeth lost was $\leq 5$.

In the severe periodontitis group (M3), the average CAL was $\geq 2.5 \mathrm{~mm}$, loci (with proximal CAL $\geq 5 \mathrm{~mm}$ ) were distributed in three to four different areas and the number of teeth lost was $\leq 14$. Examination of complete intercuspal position and six loci were probed in each tooth (buccal surface, and the distal, central and proximal lingual surfaces of the tooth) for the examination of CAL, probing depth (PD), bleeding index (BI), calculus index (CI) and plaque index (PLI). The average values of individual indexes were used as the corresponding values for each patient. Blood sampling and assays On the morning (6-7 am) of the second day of hospitalization, a blood sample was collected by drawing $3 \mathrm{ml}$ of fasting venous blood. Total cholesterol and triglycerides was determined with ELISA kits, whereas HDL-C and LDL-C were detected by the direct method. Normal ranges of blood lipid were as follows: total cholesterol, $3.10-5.18 \mathrm{mmol} / \mathrm{L}$; triglycerides 0.33-1.7 mmol/L; HDL-C, 1.09-1.63 mmol/L; and LDL-C, 1.94-3.61 mmol/L.

Statistical analysis were performed using SPSS 20.0. Continuous variables were expressed as Mean SD; categorical variables were presented as the number (percentage) of patients. The Chisquare test was used to detect any difference in severity of periodontitis; the Mann-Whitney U test was used to detect any difference in age, total cholesterol, triglyceride, HDL-C, LDL-C, BMI, Angiography Finding, CAL, PD, BI, CI, and PLI among the male and female groups. The KruskalWallis test was used to detect any difference among M0, M1, M2 and M3 groups, and when a significant difference was detected, the MannWhitney $U$ test and Bonferroni adjustment of critical p-values were used for between group comparisons. Multiple linear regression analysis was used to examine the correlations between the Angiography Finding and CAL, PD, BI, CI and PLI. The significance level a was set at 0.05.

Fig 01 ECG showing Ischemic changes

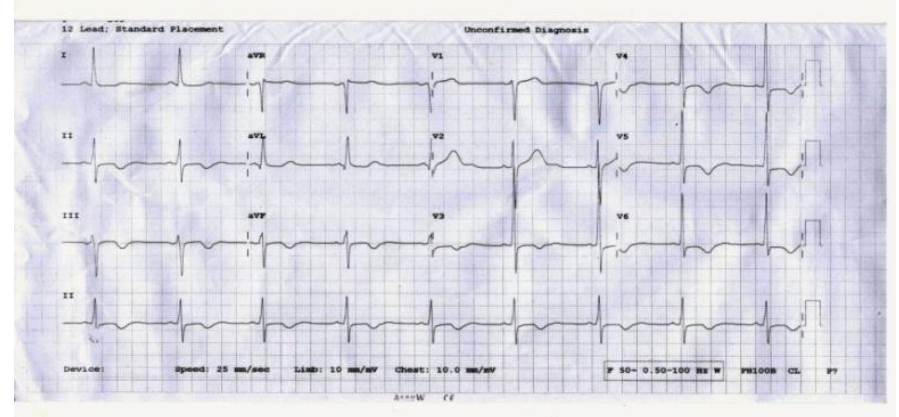

Fig 02 Showing Stenosis of the Coronary Artery (Marked black arrow)

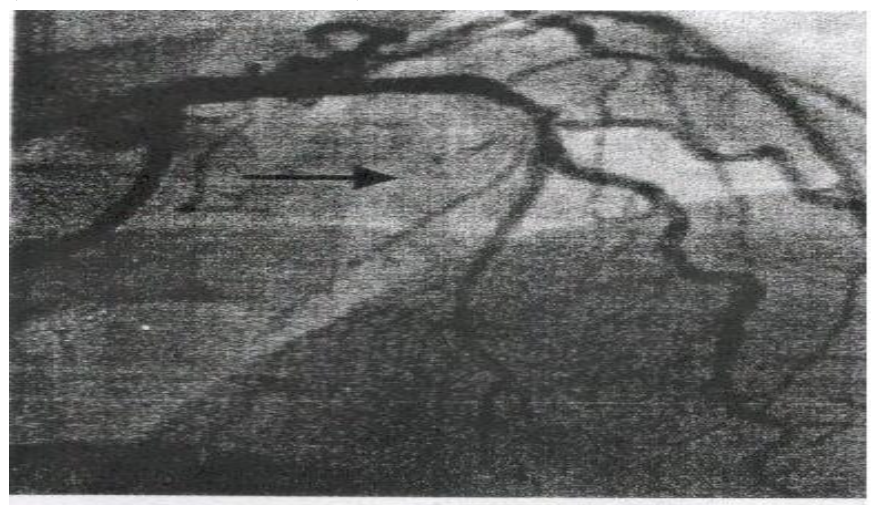

Fig 03 Showing Coronary Artery Stenosis at different level (Marked by Black Arrow)

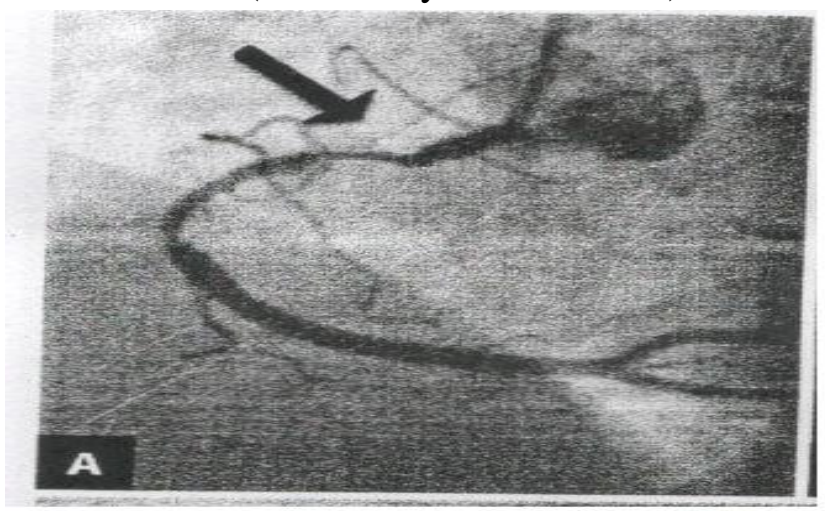




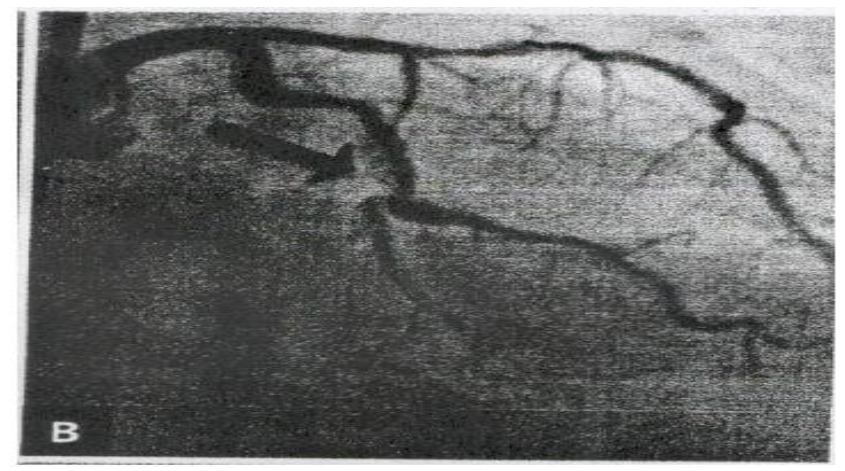

\section{Observation \& Results}

Patient demographic characteristics are shown in Table 1. A total of 350 patients with CAD were included in this study; 246 males and 104 females. There were 68 patients in the M0 group, 84 in the M1 group, 92 in the M2 group and 106 in the M3 group. The total cholesterol, triglycerides, HDL-C and LDL-C were in the normal range in all patients. The demographic characteristics of the M0, M1, M2 and M3 groups are shown in Table 2. The four groups were similar with respect to age, total cholesterol, triglyceride, LDL-C and BMI (all $\mathrm{p}>0.05$ ). Compared with the M0 group, the M1 and M3 groups had lower HDL-C, although the values are still in the normal range. The Angiography Finding was significantly higher in the M3 group compared with that in M0 group. For the severity of periodontitis, the M1, M2 and M3 groups had significantly increased CAL and CI compared with the M0 group. Furthermore, compared with the M1 group, the M2 and M3 groups had significantly increased CAL and the M3 group had a significantly increased CI. In addition, the M3 group had significantly increased CAL and CI compared with the M2 group. The PD, BI and PLI were significantly higher in the M2 and M3 groups compared with the M0 group, and the BI in the M3 group showed a significant increase compared with that in the M1 group. The Angiography Finding among all the patients stratified according to the severity of periodontitis. The correlation between the severity of CAD and CAL, PD, BI, CI and PLI, Multiple regression analysis revealed that PD and PLI were significantly correlated with the Angiography Finding. After adjusting for CAL, PD, BI, CI and PLI, an increase in PD of 1 unit was associated with an increase in the Angiography Finding of 0.62 points. Furthermore, an increase in PLI of 1 unit was associated with an increase in the Angiography Finding of 0.57 points.

Table 01: Clinical Characteristics

\begin{tabular}{|l|c|c|}
\hline S. No. & Variable & Mean \pm SD \\
\hline 1 & Age (years) & $65.0 \pm 4.6$ \\
\hline 2 & Total cholesterol $(\mathrm{mmol} / \mathrm{l})$ & $4.3 \pm 1.3$ \\
\hline 3 & Triglyceride $(\mathrm{mmol} / \mathrm{l})$ & $1.7 \pm 0.8$ \\
\hline 4 & HDL-C $(\mathrm{mmol} / \mathrm{l})$ & $1.3 \pm 0.2$ \\
\hline 5 & LDL-C $(\mathrm{mmol} / \mathrm{l})$ & $2.8 \pm 1.4$ \\
\hline 6 & BMI $(\mathrm{kg} / \mathrm{m} 2)$ & $25.3 \pm 3.1$ \\
\hline 7 & Gender n $(\%)$ & Both \\
\hline 8 & Male & $246(70.2)$ \\
\hline 9 & Female & $104(29.8 \%)$ \\
\hline 10 & Severity of periodontitis n $(\%)$ & $68(19.42 \%)$ \\
\hline 11 & M0 & $84(24.00 \%)$ \\
\hline 12 & M1 & $92(26.28 \%)$ \\
\hline 13 & M2 & $106(30.28 \%)$ \\
\hline 14 & M3 & \\
\hline
\end{tabular}

Table 02: Severity of Periodontitis among all patients along with clinical characteristics

\begin{tabular}{|l|l|c|c|c|c|c|}
\hline S. No. & Variable & M0 & M1 & M2 & M3 & p-value \\
\hline 1 & Age (years) & $62.2 \pm 2.9$ & $64.6 \pm 2.3$ & $65.5 \pm 2.2$ & $64.1 \pm 5.9$ & 0.171 \\
\hline 2 & Total cholesterol (mmol/l) & $4.1 \pm 1.5$ & $4.6 \pm 0.8$ & $4.2 \pm 1.7$ & $3.9 \pm 1.4$ & 0.512 \\
\hline 3 & Triglyceride (mmol/l) & $2.1 \pm 1.6$ & $1.3 \pm 0.7$ & $1.7 \pm 1.3$ & $1.9 \pm 0.8$ & 0.832 \\
\hline 4 & HDL-C (mmol/l) & $1.2 \pm 0.2$ & $1.0 \pm 0.2^{*}$ & $1.1 \pm 0.2$ & $1.1 \pm 0.4^{*}$ & $0.006 \S$ \\
\hline 5 & LDL-C (mmol/l) & $2.2 \pm 0.8$ & $2.2 \pm 0.7$ & $2.1 \pm 1.3$ & $2.5 \pm 1.4$ & 0.768 \\
\hline 6 & BMI (kg/m2) & $21.3 \pm 5.3$ & $24.2 \pm 3.9$ & $23.8 \pm 3.4$ & $24.4 \pm 2.6$ & 0.587 \\
\hline 7 & Angiography Finding & $10.9 \pm 12.8$ & $15.2 \pm 17.8$ & $31.2 \pm 35.0$ & $36.0 \pm 36.7^{*}$ & $0.003 \S$ \\
\hline 8 & Periodontitis Index & & & & & \\
\hline 9 & CAL & $0.5 \pm 0.2$ & $1.4 \pm 0.3^{*}$ & $2.2 \pm 0.5^{*} \dagger$ & $3.2 \pm 0.6^{*} \dagger \dagger$ & $<0.0001 \S$ \\
\hline 10 & PD & $2.6 \pm 0.3$ & $3.6 \pm 0.5$ & $3.3 \pm 0.7^{*}$ & $3.4 \pm 0.7^{*}$ & $<0.0001 \S$ \\
\hline 11 & BI & $1.1 \pm 0.7$ & $1.4 \pm 0.6$ & $1.7 \pm 0.6^{*}$ & $2.8 \pm 0.7^{*} \dagger$ & $<0.0001 \S$ \\
\hline 12 & CI & $1.8 \pm 0.5$ & $1.6 \pm 1.0^{*}$ & $1.2 \pm 0.7^{*}$ & $2.7 \pm 0.8^{*} \dagger \dagger$ & $<0.0001 \S$ \\
\hline 13 & PLI & $1.6 \pm 1.0$ & $2.9 \pm 0.8$ & $2.1 \pm 0.5^{*}$ & $2.4 \pm .3^{*}$ & $<0.0001 \S$ \\
\hline
\end{tabular}

§Significant difference among the four periodontitis groups.

*Significant difference between M0 and M1/M2/M3.

$\dagger$ Significant difference between M1 and M2/M3.

†Significant difference between M2 and M3.

HDL-C = high density lipoprotein cholesterol; LDL-C = low density lipoprotein cholesterol; GS = Gensini score;

$\mathrm{CAL}=$ clinical attachment loss; $\mathrm{PD}=$ probing depth; $\mathrm{BI}=$ bleeding index; $\mathrm{CI}=$ calculus index; $\mathrm{PLI}=$ plaque index. 


\section{Discussion}

The aim of the present study was to find out the association between periodontitis and CAD \& it's outcomes over 1 years in patients of CAD, \& to find out whether the presence of periodontal status worsen CAD. In our study found out that there is correlation between periodontitis \& coronary artery disease.

The results showed that the Angiography finding was significantly higher in the M3 group with the most severe periodontitis compared with the M0 group with no peridontitis/gingivitis. Furthermore, multiple linear regression analysis using all patients showed that PD and PLI were positively correlated with the Angiography finding. These results indicate that periodontitis might be an independent risk factor for the severity of CAD. An important aspect of the present study was that it was conducted in a relatively homogenous population.

Even though previous studies have used CAL to classify peridontitis, other studies have used pocket (probing) depth. There is a lack of consensus on the best definition. ${ }^{6}$ When patients in the present study were stratified into four groups using the Armitage method to define the severity of periodontitis, the M3 group with the most severe periodontitis had a significantly higher Angiography finding than the M0 group with no periodontitis/gingivitis.

The mechanism for this is not clear, there may have been complex relationships among the variables that were important in the multivariate model. A major strength of this study was that the degree of coronary artery stenosis was assessed in 14 different segments of the coronary arterial tree using CAG. The Angiography system assigned different weighting factors to the 14 segments and generated a single score that reflected the overall extent and severity of coronary stenosis in each patient.

Although other studies have used CAG to document the presence of coronary artery stenosis in patients with periodontitis, ${ }^{8-9}$ our study is the first to show a relationship between the extent \& severity of coronary stenosis throughout the coronary arterial tree \& the severity of periodontitis. An interesting finding in the present study was that $17 \%$ of patients with CAD documented by CAG had no periodontitis/ gingivitis. $\mathrm{CAD}$ is a multi factorial disorder but it has got a strong correlation between the group (M0) was not significantly different from that in the M1 group with mild periodontitis. These results indicate that there are multiple factors that lead to CAD and this may well account for the lack of a strong association between periodontitis. When regression analysis was conducted separately in each gender, there was no significant association between the severity of periodontitis and CAD (data not shown). This may well have been due to the reduction in statistical power due to sample size. It is possible that gender has an influence on the association between periodontitis and CAD, but this will need to be evaluated in a larger population. The results of our study indicated that periodontitis might be an independent risk factor for $\mathrm{CAD}$ in the population $\geq 60$ years old. Persson et al. ${ }^{7}$ showed a correlation between alveolar bone loss (assessed by radiography) and calcium accumulation in the carotid arterial wall that ranged in age from 60-75 years old. Aalveolar bone loss was associated with a self-reported history of heart attack in similar study. Thus, the results of our study tend to confirm these previous findings that there is an important association between periodontitis and CAD in patients $\geq 60$ years old. This study has several important limitations. The number of volunteers recruited in this study without receiving medication for $\mathrm{CAD}$ was small, the design was showing \& found no control group. Furthermore, our study population was limited to patients $\geq 60$ years old. The relationship between periodontitis and CAD may be different in more ethnically diverse populations or younger patients or patients with more risk factors. To clearly establish the relationship between periodontitis and CAD, unified standards should be formulated and a larger sample number should be included. In 
addition, to further clarify the effect of periodontitis on coronary artery stenosis, CAD patients with periodontitis should receive periodontal treatment and be followed in a longitudinal manner to determine whether periodontitis treatment improves the prognosis of CAD patients.

\section{Conclusions}

The extent and severity of coronary artery stenosis in patients $\geq 60$ years old with CAD is positively correlated with the severity of periodontitis \& it might be an independent risk factor \& should always be considered in all patient who present with coronary artery disease having periodontitis if controlled then outcome may be better in long term, if not immediate.

\section{References}

1. Smith SC Jr, Greenland P, Grundy SM. AHA Conference Proceedin Angiography finding. Prevention Conference V. Beyond secondary prevention: identifying the high-risk patient for primary prevention: executive summary. American Heart Association. Circulation 2000;101:111116.

2. Makris CG, Makris MC, Wilmot W, Geroulakos G, Falagas ME. The role of infection in carotid plaque pathogenesis and stability: the clinical evidence. Curr Vasc Parmacol 2010;8:861-872.

3. Bahekar AA, Singh S, Sandeep S, Molnar $\mathrm{J}$, Arora R. The prevalence and incidence of coronary heart disease is significantly increased in periodontitis: a meta-analysis. Am Heart J 2007; 154:830-837.

4. Kebschull M, Demmer RT, Papapanou PN. 'Gum Bug, Leave My Heart Alone!'-Epidemiologic and mechanistic evidence linking periodontal infections and atherosclerosis. J Dent Res 2010;89:879-902.
5. Gensini GG. A more meaningful scoring system for determining the severity of coronary heart disease. Am J Cardiol 1983; 51:606.

6. Savage A, Eaton KA, Moles DR, Needleman I. A systematic review of definitions of periodontitis \& methods that have been used to identify this disease. $\mathbf{J}$ Clin Periodontol 2009;36:458-467.

7. Persson RE, Hollender LG, Powell VL, et al. Assessment of periodontal conditions and systemic disease in older subjects. II. Focus on cardiovascular diseases. J Clin Periodontol 2002;29:803-810.

8. Couper DJ, Beck JD, Falkner KL, et al. The Periodontitis and Vascular Events (PAVE) pilot study: Recruitment, retention, and community care controls. J Periodontol 2008;79:80-89.

9. Offenbacher S, Beck JD, Moss K, et al. Results from the Periodontitis \& Vascular Events (PAVE) Study: A pilot multicentered, randomized, controlled trial to study effects of periodontal therapy in a secondary prevention model of cardiovascular disease. J Periodontol 2009;80:190-201. 